\title{
USING LEARNING THEORIES FOR EDUCATION ON THE WEB
}

\author{
A Web Application for Teaching and Learning \\ Computer Networks
}

\author{
Evandro Cantú \\ Federal Center of Technological Education of Santa Catarina \\ São José, SC, Brazil \\ cantu@sj.cefetsc.edu.br
}

Jean Marie Farines

Department of Automation and Systems

Federal University of Santa Catarina

Florianópolis, SC, Brazil

farines@das.ufsc.br

José André Angotti

Department of Education

Federal University of Santa Catarina

Florianópolis, $S C$, Brazil

angotti@ced.ufsc.br

\begin{abstract}
The objective of this paper is to present a Web application, constructed in order to help and guide teachers and learners of computer networks. This application defines a context for teaching and learning which is based on a methodological approach stemmed from some modern educational theories. Concept maps, a graphical representation of the relationships among concepts, are used to model and organize the knowledge of the application domain, in our case, computer networks. This application offers a very concise form to present the concepts and principles to be taught or learned and consequently to organize the educational process. Guidelines for using this application and its associated environment are also presented.
\end{abstract}

Keywords: Technological Education, Concept Maps, Curriculum Organization, Computer Networks Teaching and Learning 


\section{Introduction}

One of the most important skills required by the new engineering profile is the capability to acquire knowledge in a continuous and incremental way. In particular, the fast technological changes require a constant improvement in technological education.

In this paper, we choose computer networks as a good example of a domain with a continuous change of the technological contents. Furthermore, the high complexity and the great diversity of the contents related to computer networks is another question to be solved for the development of learning.

To better develop this kind of courses, alternative methodologies must be considered in the curriculum organization and implementation. These methodologies need to deal with the learning of the underlying concepts of the domain and, at the same time, the preparation of the students for their professional life, in a world with a continuous process of technological changes.

In this context, we present some methodological guidelines and a Web application to improve the teaching and learning of technological courses. Our methodological approach combines the generative themes, proposed by the Brazilian educator Freire, 1981, and the assimilation theory and concept maps, proposed by Ausubel et al., 1980.

In the next sections we present the principal contributions of educational researches used in this work and the concept maps. After that, we discuss the knowledge representation applied to computer networks domain. Finally, we present the structure of the Web application proposed in this work, showing how teachers and learners of computer networks course can use this application.

\section{Methodological Considerations}

The Brazilian educator Freire, 1981, brought some important contributions for the educational process. His ideas have been applied in general education, including also science and physics education (Delizoicov et al., 2003). According to Freire, 1981, the learning activities must be developed around generative themes that are part of the students cultural environment. The generative themes increase the motivation of students and allow them to extend their knowledge about the subject, including social and political factors that can contribute to form complete citizens with critical minds.

If we take our domain of interest, the computer networks, we can see that the Internet and its applications got into the homes and businesses of millions of people worldwide, changing the peoples way of life. So, the 
Internet can be used as a key generative theme to anchor the educational process.

The generative themes present a rupture in the way of curricula have been elaborated, which are, normally, strongly based on contents, organized in a rigid and systematic manner. In a dynamic domain, with a lot contents, like computer networks, the use of generative themes is a good criteria for helping in the selection of contents.

To use these ideas is important to relate the generative themes with the fundamental concepts that allow giving a global vision of the theme.

The assimilation theory, presented by Ausubel et al., 1980, can help in the this process. This educational theory describes how the students acquire concepts and how these concepts are organized in their minds. For the assimilation theory, the learning occurs when a new information is obtained from a planned effort by the learner to link this information with some relevant concepts already existing in his/her cognitive structure. To accomplish this task, the suggestion is to start the learning from more general and comprehensive concepts and then, to go towards more specific ones.

To use the assimilation theory, one important task is to determine the conceptual structure of the domain to be taught. In other words, we have to identify the underlying concepts and principles of the domain and hierarchically organize them, from the more general and inclusive concepts towards the more specific ones. Afterwards, this conceptual structure can be used to put the learning in sequence.

The concept maps, developed by Novak, 2003, in the sequence of researches about the assimilation theory, can help in this task.

The concept maps are a kind of graph used to represent the relationships among a group of concepts. To each node of the graph a concept can be associated. The labelled links between nodes show the relationships among concepts. The figure 1 and 2 are examples of concept maps, the first shows a brief picture of the computer networks evolution, and the second shows a global vision of the Internet and its principal services.

In the concept map representation, concepts are represented in a hierarchical way with the most general and inclusive concepts at the top and the more specific ones organized hierarchically below. For a concept corresponding to a node, it is also possible to build another concept map with the objective of refining it.

Concept maps have already been used for a variety of educational purposes (Novak, 2003; Cañas et al., 2003). The concept maps can be helpful to clearly present the material to be learned, identifying the large general concepts prior to instruction and assisting in the sequencing of learning through progressive and more specific and explicit knowledge. 
Based on these educational contributions, we intent to use generative themes, to anchor the curriculum, and to model the knowledge of the domain to be taught or learnt using concept maps.

In the next section we discuss the knowledge representation of computer networks domain and the use of concept maps.

\section{Knowledge Representation In Computer Networks Domain}

In a traditional teaching approach, the curriculum is organized by following the classical structure of the programmatic contents of a course in a rigorous way. The contents are previously divided into topics and presented sequentially and in depth to the students. Normally, the teaching is based on textbooks, which are used by teachers as the principal didactic material.

In a domain with a continuous change of the technological contents, as are the computer networks domain, this approach has some limitations. The great question is how to select and organize the contents to be taught.

To organize the contents, many computer networks textbooks divide the chapters based on the layers of network architecture, like in Tanenbaum, 2003. This way of organization largely influenced the teachers of computer networks during the last years. Normally, the courses were strongly based on the contents, with each topic or protocol being studied in depth as an individual component. A common approach for explore the contents was cover the layers in a bottom-up manner, following the book organization, starting from the physical medias and finishing with the network applications. Frequently, the network applications were not discussed due to the lack of time and also because they were considered less important in the point of view of the network developers.

The rigid pedagogical material of textbooks, associated with the high complexity and the great diversity of the contents, do not motivated the students, imposing great difficulties for they understand concepts and relate the topics under study with real applications.

Kurose and Ross, 2000, innovated with "a top-down approach featuring the Internet", which begins at the application layer and works its way down the layers. According to the authors, the top-down approach has several important benefits. It places emphasis on the application layer, which is the high growth area of computer networks. It is a powerful approach to motivate students. It enables instructors to introduce network applications development at an early stage. 
Starting with the use of networks and global behavior we can affirm that we are closer to the ideas of generative themes of Freire, 1981, which increase motivation and give an explicit meaning to the knowledge taught.

However, inverting only the way of exploring the layers is not sufficient. It is also interesting to explore the concepts and technologies in a general-to-specific manner, in order to facilitate acquisition of fundamental concepts by the learners.

The importance of the fundamental concepts is also emphasized by Peterson and Davie, 2000, which is loosely organized their textbook around the traditional network layers. The authors propose to "start with the first principles and walks through the thought process that led to today's networks". They say that in a dynamic and changing domain as computer networks, "the most important thing a textbook can offer is perspective to distinguish what's important and what's not, between what's lasting and what's superficial".

However, this approach can also be enriched organizing the concepts in a hierarchical manner, from the general-to-specific concepts, placing each one in the global view of networks.

Despite the development of computer networks, when we analyze its historical evolution, we can see that many fundamental concepts remain unchanged. The concept map of figure 1 shows some fundamental concepts of computer networks throughout time. Once you understand these concepts, any new problem or technology will be relatively easy to apreciate. So, a reasonable approach for a computer networks course should focus on the current networks technologies and search for the fundamental concepts which allow the understanding of these technologies.

Different from classical computer networks textbooks, our approach try to start the study with current network applications and technologies, like the Internet and Local Area Networks, selecting its underlying principles, and exploring its in a general-to-specific manner.

Based on a concept map representation, the idea is to start from a wide vision of a problem or a theme, looking firstly to its big picture, its major parts and the relationship among those parts. After, a part (or a detail) can be examined, without losing the global view. Then, in any time, one can go back to the wide vision to review other parts of the whole problem or topic and to examine the context of certain parts within the whole. Chou, 1999 neatly compare this approach with a photograph using a zoom lens.

For example, suppose that someone wants to teach the topic reliable data transfer, offered by the connection oriented service of the Internet protocol TCP. The first thing to do is to place the topic in hand into 


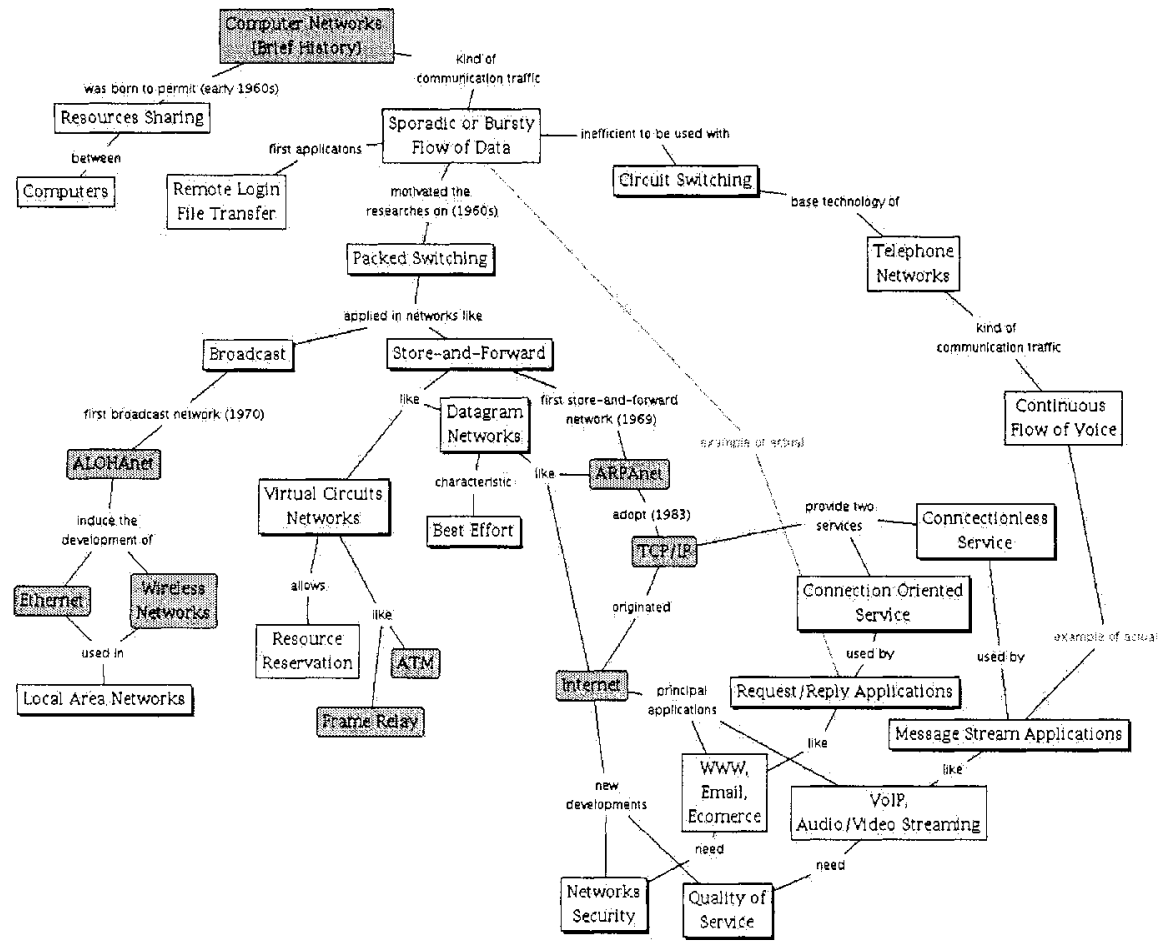

Figure 1. A brief picture of computer networks evolution

the global view of the Internet technology. The concept map of figure 2 shows a manner of doing it, showing how each concept are related with each other and its role in the global behavior of the Internet.

After that, the connection oriented service of TCP protocol can be detailed, as show the figure 3 , where the main concepts related to a reliable data transfer are taken into account.

These concept maps (figure 2 and 3 ) can be used to structure the learning process. They are examples of how concept maps are incorporated in a Web application presented in the following.

\section{Educational Framework For Teaching And Learning On The Web}

In this section we present a Web application, constructed in order to help and guide teachers and students to organize and improve their activities. 


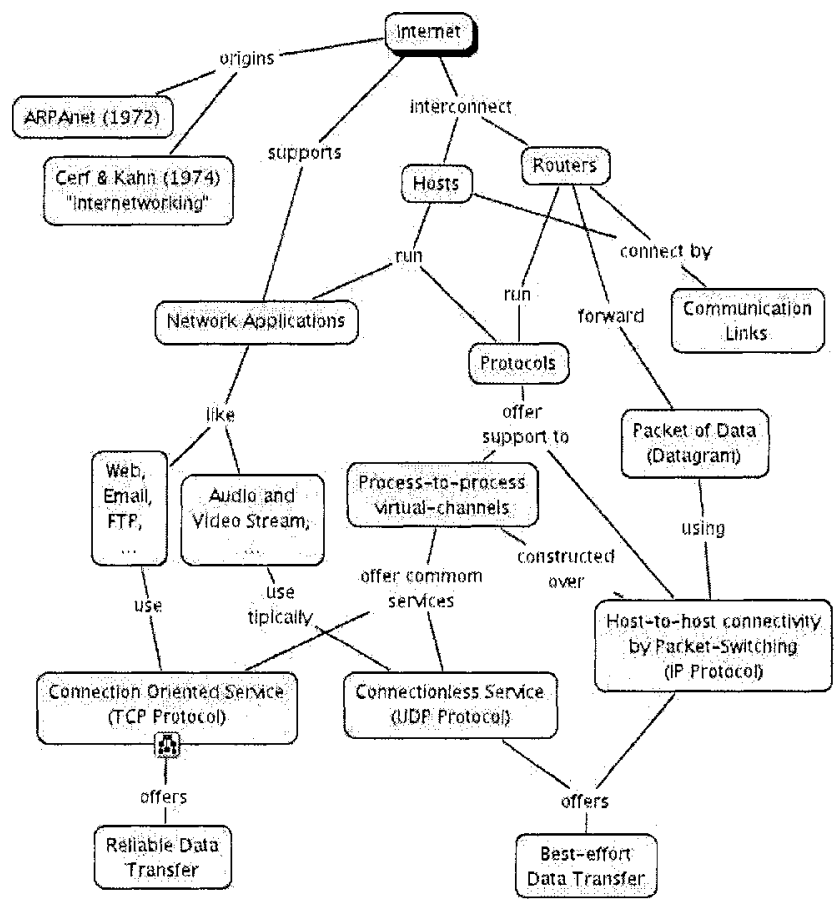

Figure 2. Vision of principal Internet services

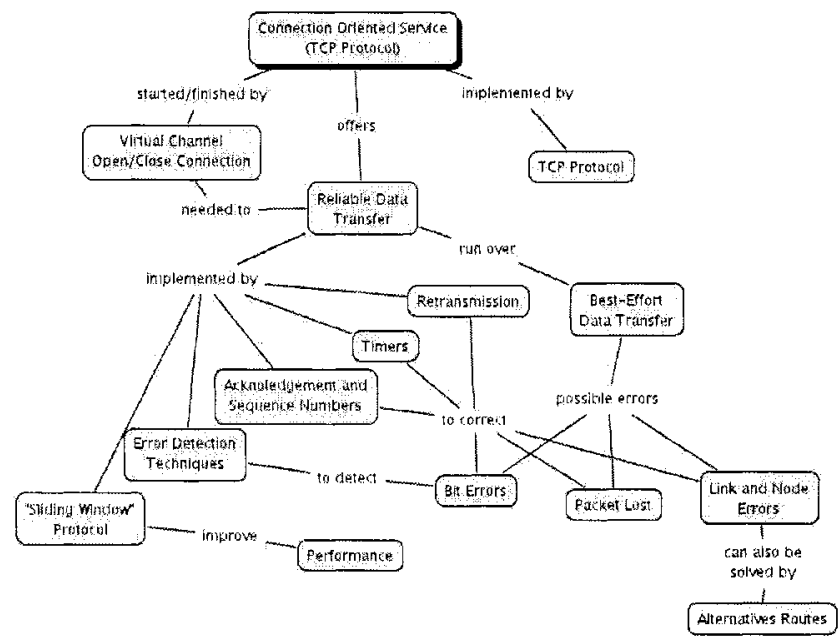

Figure 3. The problem of reliable transmission 
The concept map in figure 4 represents the roles and the composition of each part of this application.

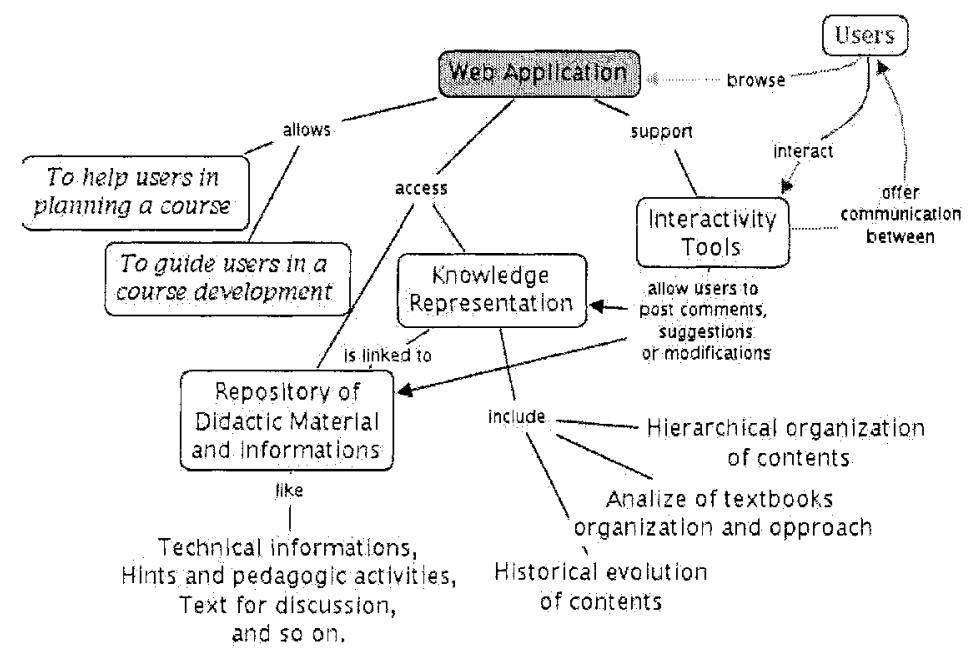

Figure 4. Roles and composition of the educational framework

The users can browse on the Web environment, searching for information, getting help in planning a specific course in computer networks domain or to be guided during the development of a course.

The environment can provide access to a knowledge representation of the technological domain, constructed by concept maps, associated with didactic materials and other storage information. Special links can be assigned to each concept in a concept map to highlight other elements of the concept, including texts, pictures, technical references, access to other related Web sites and so on.

Users can also interact with the application by means of tools that allow interactivity with the system and also with others users. It's possible for users to post suggestions or comments about the information on the Web, to access frequent asked questions, and to discuss with other users about specific themes.

The Web environment is being constructed using educational tools. The IHMC Cmap Tool ${ }^{1}$ is being used to build the concept maps. The tool also permits us to export the concept maps to HTML format to be accessed in a Web environment.

By using the environment, the application can help educators in planning a course offering general options of concept maps, where topics, concepts and technologies are hierarchically organized. 
To enrich the educational process, to each main concept is associated some didactic material, making a scenario to guide the teacher in the development of learning.

Learners can also use the application to browse and search for information. The graphical and hierarchical nature of concept maps helps them to keep any concept well placed in the hierarchical structure, without losing the global view. From a concept, students can access documents, videos, simulation applet or other information related to this topic.

At the moment the development of the Web environment has arrived at a preliminary phase, where we are linking the knowledge structures constructed by concept maps to related information. We are also elaborating and systematizing didactic materials to be added into the application.

\section{Conclusion}

In this paper we presented some methodological guidelines to be applied in computer networks teaching and learning, stemming from some modern learning theories. An educational application, constructed on the Web, synthesizes our approach. Teachers and learners of computer networks can use this application in order to help and guide their work. The application is based on concept maps, a graphical representation of the relationships among concepts, used to model and organize the knowledge of the application domain. With the concept maps, the knowledge is organized in a hierarchical way, with the most general and comprehensive concepts on the top and the more specific ones, arranged bellow the map. This organization facilitates the developing of learning following a general-to-specific approach of concepts. Didactic materials are also part of the Web application and can be used by teachers in their activities.

\section{Notes}

1. IHMC Cmap Tool is a tool for construct concept maps of The Institute for Human and Machine Cognition (University of West Florida, USA). http://cmap.coginst.uwf.edu

\section{References}

Ausubel, D. P., Novak, J. D., and Hanesian, H. (1980). Psicologia Educacional. Interamericana, Rio de Janeiro.

Cañas, A. J., Ford, K. M., Coffey, J., Reichberzer, T., Suri, N., Carff, R., Shamma, D., Hill, G., Hollinger, M., and Nitrovich, T. (2003). Herramientas para construir y compartir modelos de conocimiento. Institute for Humen and Machine Cognition, University of West Florida, USA. 
Chou, C. (1999). Developing hypertext-based learning courseware for computer networks: The macro and micro stages. IEEE Transaction on Education, 42:39-44.

Delizoicov, D., Angotti, J. A., and Pernanbuco, M. M. (2003). Ensino de Ciências: Fundamentos e Métodos. Docência em Formação. Cortez, São Paulo.

Freire, P. (1981). Pedagogia do Oprimido. Paz e Terra, Rio de Janeiro.

Kurose, J. F. and Ross, K. W. (2000). Computer Networking: A Top-Down Approach Featuring the Internet. Addison Wesley.

Novak, J. D. (2003). The theory underlying concept maps and how to construct them. Institute for Humen and Machine Cognition, University of West Florida, USA.

Peterson, L. L. and Davie, B. S. (2000). Computer Networks: A System Approach. Morgan Kaufmann, San Francisco, second edition.

Tanenbaum, A. S. (2003). Computer Networks. Prentice Hall, fourth edition. 